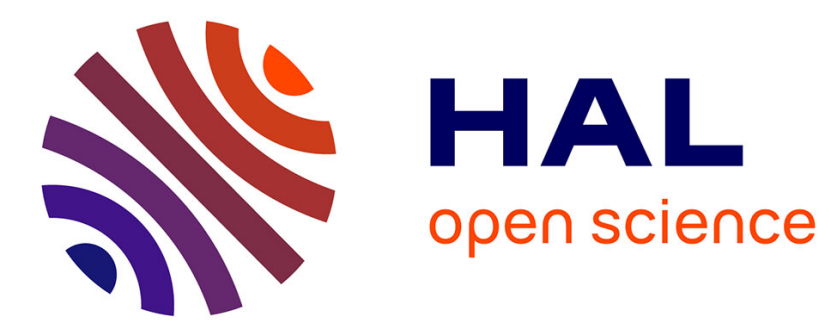

\title{
Optical imaging: Ultrafast buffering by molecular gas
} Edouard Hertz, Bruno Lavorel, Olivier Faucher

\section{To cite this version:}

Edouard Hertz, Bruno Lavorel, Olivier Faucher. Optical imaging: Ultrafast buffering by molecular gas. Nature Photonics, 2011, 5, pp.78. 10.1038/nphoton.2011.6 . hal-00564126

\section{HAL Id: hal-00564126 https://hal.science/hal-00564126}

Submitted on 5 Dec 2012

HAL is a multi-disciplinary open access archive for the deposit and dissemination of scientific research documents, whether they are published or not. The documents may come from teaching and research institutions in France or abroad, or from public or private research centers.
L'archive ouverte pluridisciplinaire HAL, est destinée au dépôt et à la diffusion de documents scientifiques de niveau recherche, publiés ou non, émanant des établissements d'enseignement et de recherche français ou étrangers, des laboratoires publics ou privés. 


\section{Optical imaging}

\section{Ultrafast buffering by molecular gas}

\section{A simple molecular gas sample can be used to achieve ultrafast optical buffering in two-dimensional optical imaging, thus serving as a promising extension of the well-developed liquid-crystal display technology.}

\section{Edouard Hertz, Bruno Lavorel and Olivier Faucher}

In the technologies surrounded us today, liquid crystals can be most found in the information display of text, images and videos. The principle of liquid crystal display (LCD) technology is based on the electro-optical Kerr effect in liquid crystals responsible for the orientation of dipole moments when exposed to an external electric field. The birefringence induced by the orientation of dipole moments is used to control the intensity of the light passing through the LCD, and therefore the switching time of the LCD are limited by the reorientation of the macromolecules that occurs in the millisecond time scale. On the other hand, the quest for large bandwidth, in response to a growing demand for high-speed data storage and communication networks, seeks for faster "alloptical" device solutions. The relatively slow time response makes LCD devices rather incompetent and inappropriate for ultrafast switching, gating, and imaging applications in the range of $\mathrm{GHz}$ to $\mathrm{THz}$.

Writing in Applied Physics Letters, Jian Wu and colleagues ${ }^{1}$, from East China Normal University in Shanghai, propose the use of gas-phase molecules in place of liquid crystals to overcome the high switching time of LCDs. They have successfully demonstrated ultrafast optical imaging involving optical image storage followed by periodic readout and display. In analogy to the LCD technology, the team orientate the molecules using a transient electric field of the order of few tens of femtoseconds, much shorter than the rotational period of the molecules. This is ensured by using a linearly polarized 35-fs femtosecond laser pulse as external field. In this approach, molecules can be seen as "quantum buffer memories" with a writing time limited by the pulse duration of the laser field. A second polarized femtosecond pulse accomplishes the reading process in a characteristic time also defined by its duration. The team use this ultrafast read-andwrite-molecule based memory to achieve ultrafast imaging.

The idea proposed by $\mathrm{Wu}$ et al. relies on the basic process of laser-induced molecular alignment that has been investigated by the strong laser field community over the last twenty years ${ }^{2}$. A laser field (the writing pulse) applied to a molecule induces a torque on its axis, leading to its alignment along the direction of field, as shown in Fig. 1a. In the short pulse regime, where the pulse duration must be shorter than the rotational period of the molecule, the alignment is produced, not only just after the field reaches its maximum, but also after the laser turn-off, in the form of revivals that clocks the time in terms of the rotational period $T_{R}$. The alignment revivals act as memory echoes of the information stored by the writing pulse. Since the short pulse alignment is a nonresonant process, the wavelengths of the writing and reading pulses can be chosen at convenience. In the nonlinear optics terminology, the reading pulse is related to the writing pulse through the retarded optical Kerr gate induced by the molecular alignment.

In a proof-of-principle experiment, $\mathrm{Wu}$ and co-workers showed that a twodimensional object imprinted in the transverse intensity profile of the writing pulse can be transferred to the gas sample, as shown in Fig. 1b. As the pulse propagates, each molecule exposed to the field is addressed with a field amplitude related to its respective position within the writing beam. The reading pulse, synchronized with the rotational period of the molecules, captures the information embedded in the birefringence of the molecular ensemble and restores it on a CCD camera through an imaging system.

As the experiment by $\mathrm{Wu}$ et $a{ }^{1}{ }^{1}$ is performed in air, the periods of the revivals associated with the two major components $\mathrm{N}_{2}$ and $\mathrm{O}_{2}$ correspond to one-quarter of the rotational periods 8.3 and $11.6 \mathrm{ps}$, respectively. This temporal feature, which can be 
scaled by choosing other molecules, allows reading out several times the image at a rate corresponding to these periods. The information is stored as long as the molecular coherence is preserved. In gas, the coherence is limited by the collisions undergone by the molecules, which can be modified by adjusting the pressure of the medium. Gas at atmospheric pressure will allow a memory time of few tens of picoseconds versus nanoseconds for more diluted medium. However, reducing the gas pressure will have a detrimental effect on the signal-to-noise ratio, since the write-and-read process is a coherent effect that depends on the squared number of molecules.

Another restrictive aspect of the technique is that it requires a moderately intense writing pulse. Typically, pulse intensity above $100 \mathrm{GW} \mathrm{cm}^{-2}$ is necessary to observe the nonlinear optical effect produced over few millimetres of atmospheric air with a suitable signal-to-noise ratio, requiring the use of an amplified femtosecond laser. A way to shift the previous limits is to increase the process efficiency by improving the alignment using a writing pulse of optimized temporal shape ${ }^{3}$ or a sequence of laser pulses ${ }^{4}$ (instead of a single writing pulse) that in both cases could be synthesized by a high-speed pulse shaper ${ }^{5}$. The later strategy could be further implemented inside a cavity with length (few centimetres) adjusted so as to match a multiple integer of the revivals period. Synchronous writing would hence provide a solution for extending the storage time of the molecular sample, by periodic regeneration of the alignment, together with improving the signal-to-noise ratio ${ }^{6}$.

The possibility of using atmospheric air as a buffer memory is fascinating. One can for instance envision integrating the write-and-read pulses and the optics (lens and polarizers) on a photonic circuit without space restrictions imposed by the use of a gas cell. The interaction region between the molecules and the light could be less than a millimetre, as defined by the spatial overlap of the two optical beams.

Besides two-dimensional imaging, Wu et al. also address the attractive issue of holographic imaging. As the presented technique is amplitude- and phase-sensitive, a three-dimensional object lit by the writing pulse can in principle be encapsulated in the molecules for subsequent reading. To preserve the spatial and temporal coherence, the application is however limited to translucent objects, from microscopic to macroscopic (of few centimetres) size. The results obtained by Wu et al. provide a demonstration of the effect on a time-consuming multi-shot basis. However, ultrafast single-shot image capture could be achieved, as shown in Fig. 2, by combining a reading pulse of long duration (few picoseconds) with a two-dimensional amplitude and phase characterization performed by a real-time optical waveform measurement technique ${ }^{7}$. The number of frames per seconds could be as high as the repetition rate of the femtosecond source (which is currently between 1 and $10 \mathrm{kHz}$ ), making this molecular-memory based technology very attractive for ultrafast holographic vision.

With a full analogy to LCD technology, Wu and colleagues have shown experimentally that a molecular gas sample, just like the surrounding air, can be used as ultrafast memory buffer to perform ultrafast optical imaging. The THz bandwidth accessible with this device exceeds the bandwidth of LCD technology and micro-electro mechanical systems by more than nine orders and six orders of magnitude, respectively. The market for such quantum memory based technology will go well beyond information storage, as it is also related to ultrafast three-dimensional acquisition (movies) and secured data transfer (cryptography).

Edouard Hertz, Bruno Lavorel, and Olivier Faucher are researchers at the Laboratoire Interdisciplinaire Carnot de Bourgogne (ICB), Université de Bourgogne, 21078 DIJON Cedex, France. Email: olivier.faucher@u-bourgogne.fr

\section{References}

1. Wu, J. et al. Appl. Phys. Lett. 97, 161106 (2010).

2. Stapelfeldt, H. \& Seideman, T. Rev. Mod. Phys. 75, 543-557 (2003).

3. Rouzée, A., Hertz, E., Lavorel, B. \& Faucher, O. J. Phys. B 41, 074002 (2008).

4. Leibscher, M., Averbukh, I. S. \& Rabitz, H. Phys. Rev. Lett. 90, 213001 (2003).

5. Vega, A., Leaird, D. E. \& Weiner, A. M. Opt. Lett. 35, 1554-1556 (2010). 
6. Jones, R. J., Moll, K. D., Thorpe, M. J. \& Ye, J. Phys. Rev. Lett. 94, 193201 (2005).

7. Fontaine, N. K. et al. Nature Photon. 4, 248-254 (2010). 
(a)

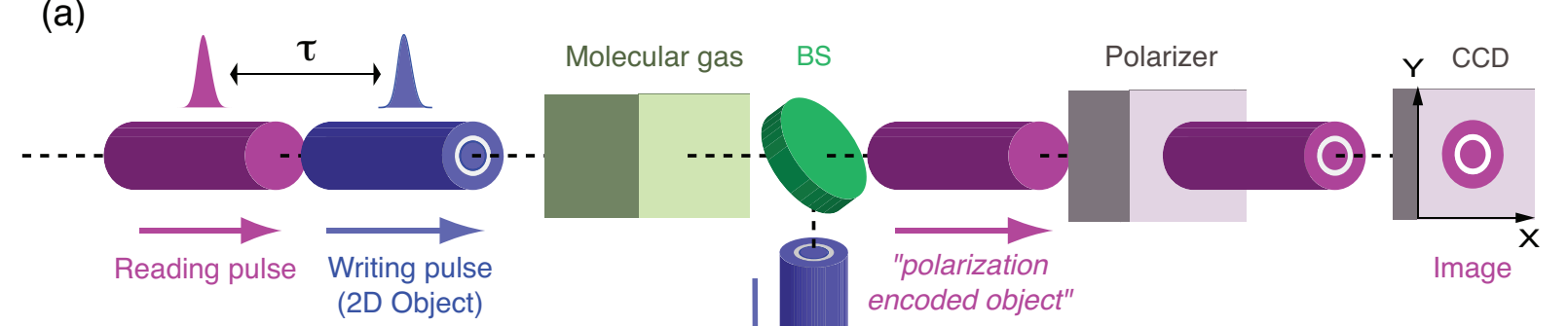

(b)

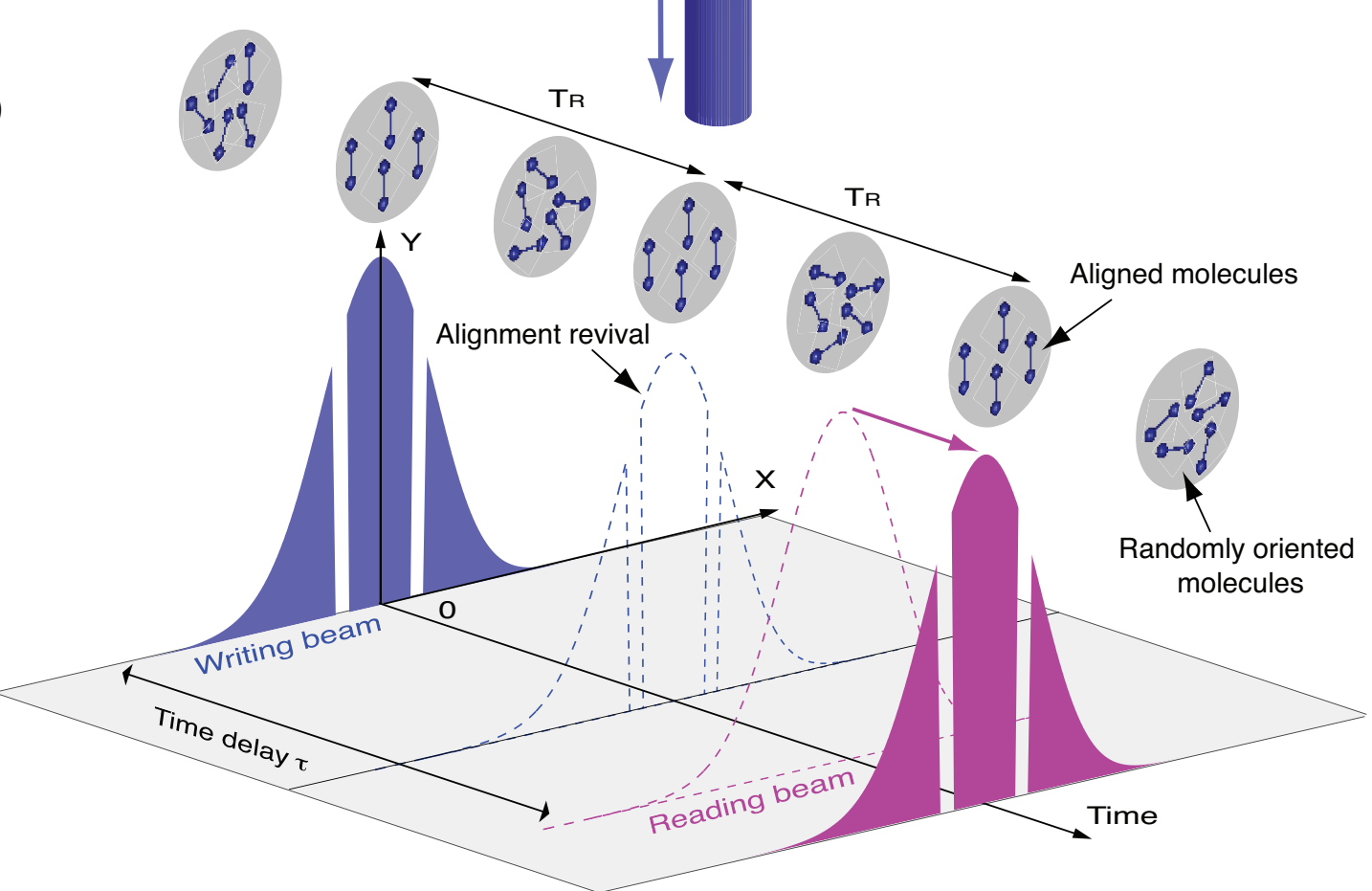

Figure 1 A two-dimensional (2D) object (the letter " $O$ ") imprinted in a writing beam is stored in the rotational response of a molecular gas sample and then restored after a time delay $\tau$ by a reading pulse. a, Schematic diagram of the experiment by Wu et $a l . b$, Birefringence left by the writing pulse within its transverse $x-y$ intensity profile. BS, beamsplitter; CCD, charge-coupled device 


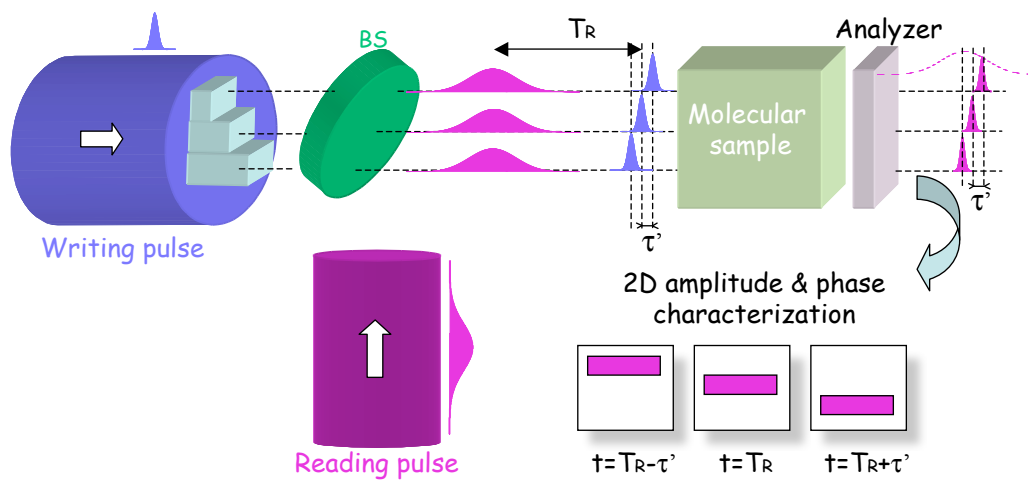

Figure 2 Schematic of single-shot holographic imaging. The writing pulse, delayed by the transparent object, initiates the alignment at different time. The reading pulse sent at time $T_{R}$ reads the thickness-dependent birefringence. The two-dimensional measurement of the temporal profile of the birefringence signal passing through the polarizer reveals the shape of the object. 